\title{
The acoustic insulation performance of infinite and finite locally resonant metamaterial and phononic crystal plates
}

\author{
Lucas Van Belle ${ }^{1,2, *}$, Claus Claeys ${ }^{1,2}$, Elke Deckers ${ }^{1,2}$, and Wim Desmet $^{1,2}$ \\ ${ }^{1}$ KU Leuven, Department of Mechanical Engineering, Celestijnenlaan 300, 3001 Leuven, Belgium \\ ${ }^{2}$ DMMS lab, Flanders Make
}

\begin{abstract}
The bending wave stop band behavior of locally resonant metamaterial and phononic crystal plates can strongly improve the vibration performance of flexible plates in targeted frequency ranges. Their acoustic insulation performance, however, is found to strongly depend on the stop band mechanism, also influencing the frequency range of improvement. While sound transmission loss improvements are predicted for both infinite periodic plates, the finite phononic crystal plate performance can differ significantly.
\end{abstract}

\section{Introduction}

Materials with stop band (SB) behavior have emerged as possible lightweight solutions with favorable noise and vibration suppression performance in targeted frequency ranges. By creating SBs for the bending waves, the sound transmission loss (STL) of flexible plates can be strongly improved. Different mechanisms can lead to SB behavior, distinguishing mainly between resonance SBs in locally resonant metamaterials (LRMs) and interference $\mathrm{SBs}$ in phononic crystals (PCs) [1]. The resonance SBs arise from Fano-type interference between incoming and re-radiated waves by sub-wavelength resonant additions. The interference SBs rely on Bragg scattering resulting from periodicity. While the STL of LRM plates has received increasing interest [2-6], PC plates for STL improvements are less investigated [7]. Besides, the STL of LRM and PC plates is often only analyzed for infinite periodic structures, discarding finite structure effects. This work investigates the STL of infinite and finite LRM and PC plates, consisting of a plate host structure with periodic spring-mass resonators and point mass additions, respectively.

\section{Methodology}

To analyze the SB behavior of the infinite periodic PC and LRM plates, dispersion curves are calculated by considering a single UC in combination with Bloch-Floquet (BF) periodicity boundary conditions (BCs) [8], assuming 2D planar periodicity with spatial period $L_{x}$ and $L_{y}$ in the $x y$ plane. The UC is represented by a Finite Element (FE) model, with following time harmonic equations of motion:

$$
\left(\mathbf{K}-\omega^{2} \mathbf{M}\right) \mathbf{q}=\mathbf{F},
$$

with frequency $\omega$, stiffness and mass matrices $\mathbf{K}$ and $\mathbf{M}$ and generalized displacements and forces $\mathbf{q}$ and $\mathbf{F}$. Apply-

*e-mail: lucas.vanbelle@kuleuven.be ing $\mathrm{BF}$ periodicity $\mathrm{BCs}$ results in a dispersion eigenvalue problem in $\omega$ and propagation constants $\left(\mu_{x}, \mu_{y}\right)$ [8]:

$$
\mathbf{A}\left(\omega, \mu_{x}, \mu_{y}\right) \mathbf{q}^{(r e d)}=\mathbf{0},
$$

with $\mathbf{q}^{(\text {red })}$ a reduced set of generalized displacements and A a matrix depending on $\omega$ and $\left(\mu_{x}, \mu_{y}\right)$. Dispersion curves for free wave propagation are obtained by imposing a set of real $\left(\mu_{x}, \mu_{y}\right)$ along the Irreducible Brillouin Contour (IBC) and solving to real $\omega$ [1].

The STL of the infinite periodic structure is predicted using the hybrid Wave Based - Finite Element (WB-FE) UC method [9], coupling the structural FE UC model to two semi-unbounded periodic acoustic WB domains, above and below the structure. The upper acoustic domain is excited by an impinging acoustic plane wave. Using a direct hybrid WB-FE coupling strategy, a system of equations is obtained, which is solved for the nodal degrees of freedom in the FE domain and the wave function contributions in the WB domain. The latter are used to calculate transmission coefficients $\tau$ and thus the STL $=$ $-10 \log _{10}(|\tau|)$ of the infinite periodic structure.

For the finite structure, an FE model of an $n \times m$ UCs sized finite plate with simply supported BCs is considered. The vibration attenuation is analyzed using a structural model, excited by a point force in the out-of-plane direction at an off-center location. For the STL prediction, the plate is excited by an incident acoustic plane wave and a diffuse field. The structure is strongly coupled to two semi-unbounded acoustic FE domains, one above and one below the structure, modeled using one layer of 3D acoustic FE elements and Automatically Matched Layer BCs to approximate the Sommerfeld radiation condition [10].

\section{LRM and PC plate design}

The LRM consists of a $1 \mathrm{~mm}$ thick aluminum plate $(E=$ $70 \mathrm{GPa}, \rho=2700 \mathrm{~kg} / \mathrm{m}^{3}, v=0.3$ ) with spring-mass res- 
onators tuned to $f_{\text {res }}=500 \mathrm{~Hz}$ and adding $80 \%$ mass, attached on a $0.05 \times 0.05 \mathrm{~m}$ periodic sub-wavelength scale. The PC plate consists of the same host structure with $0.05 \times 0.05 \mathrm{~m}$ periodically spaced point mass additions, with the same $80 \%$ mass addition. Both UCs (Figure 1) are discretized using FEM, with $10 \times 10$ shell type elements.

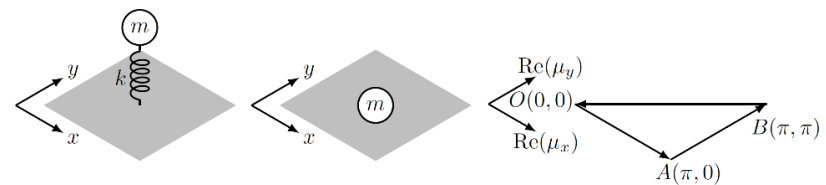

Fig. 1. UC of the infinite periodic LRM (left) and PC (center) plate and corresponding IBC (right).

\section{Dispersion curves}

For the LRM plate, a resonance based SB is obtained for the acoustically relevant out-of-plane bending waves between $445-644 \mathrm{~Hz}$, around $f_{\text {res }}$ (Figure 2a). For the PC plate, an interference based bending wave SB is obtained between 898-976 Hz near the Bragg interference limit at $968 \mathrm{~Hz}$ (Figure 2b). For the same mass addition, the LRM plate achieves a wider SB at lower frequencies.

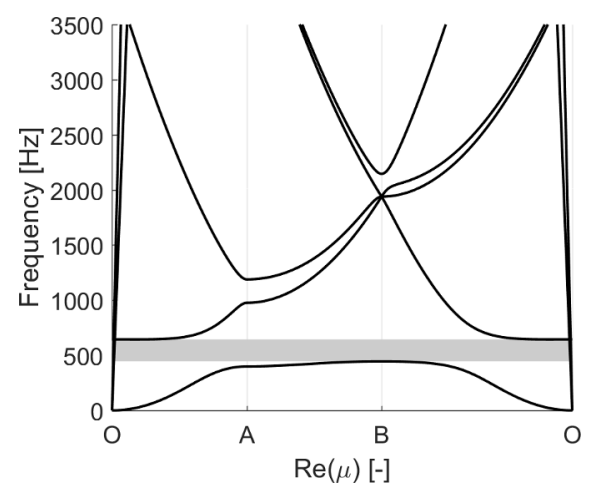

(a)

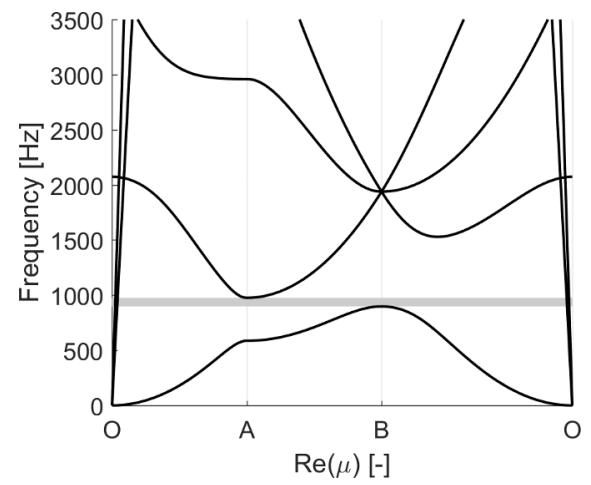

(b)

Fig. 2. Dispersion curves along the IBC for the LRM (a) and PC (b) plate UC.

\section{Finite plate vibration attenuation}

The vibration attenuation of both the LRM and PC plates is verified with a $0.6 \times 0.45 \mathrm{~m}$ finite plate with simply supported BCs, excited by a unit point force in the out-ofplane direction at an off-center location $(0.295,0.22) \mathrm{m}$. The RMS average plate displacement of both the LRM and PC plates shows a distinct frequency range of attenuation and without structural modes, corresponding to the predicted SBs (Figure 3). The vibration attenuation inside the LRM plate SB is stronger as compared to the PC plate.

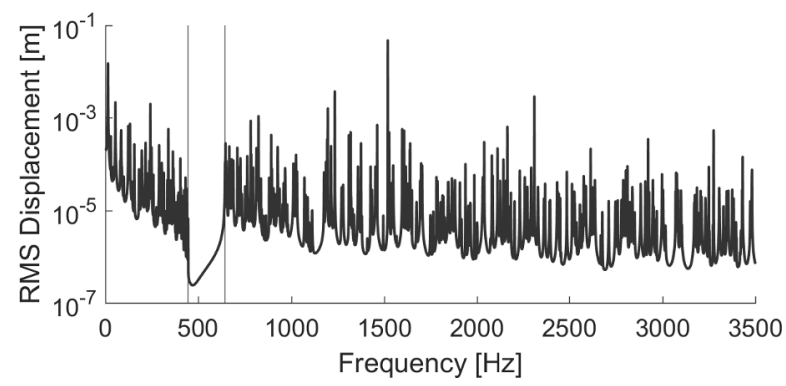

(a)

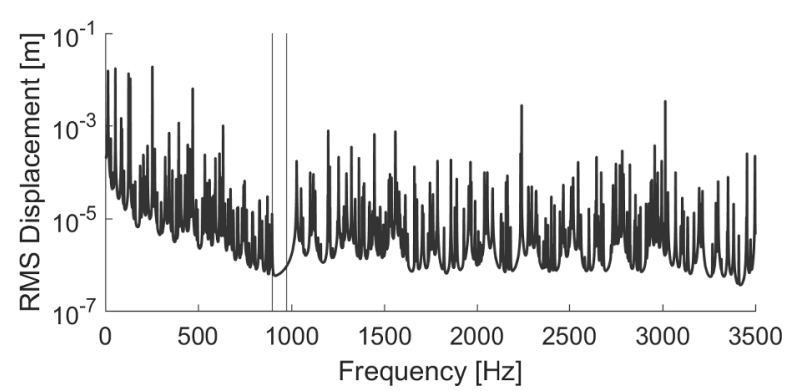

(b)

Fig. 3. RMS average plate displacement of the finite LRM (a) and PC (b) plate subjected to a point force, with SB (vertical lines).

\section{Infinite plate STL}

The hybrid WB-FE UC method is applied to the FE UCs to calculate the STL of the infinite periodic structures coupled to acoustics $\left(c=340 \mathrm{~m} / \mathrm{s}, \rho=1.225 \mathrm{~kg} / \mathrm{m}^{3}\right)$, for normal plane wave incidence (Figure 4 ). The LRM plate STL shows a peak at $480 \mathrm{~Hz}$ inside the SB near $f_{\text {res }}$, followed by an STL dip at $644 \mathrm{~Hz}$ resulting from an additional lowfrequency coincidence zone caused by the repeated bending wave mode after the SB [11]. The LRM plate outperforms the acoustic mass-law around the STL peak, compared to a $1 \mathrm{~mm}$ homogeneous plate with bare host structure mass density $\rho_{\text {host }}$ and equivalent static mass density $1.8 \rho_{\text {host }}$. The LRM plate STL tends to the STL of the $1 \mathrm{~mm}$ homogeneous plate with mass density $1.8 \rho_{\text {host }}$ and $\rho_{\text {host }}$ respectively before and after the SB, caused by the in-phase and anti-phase motion between resonators and host structure before and after the SB. The PC plate STL also shows 
a peak-dip behavior, with an STL peak at $1628 \mathrm{~Hz}$ outperforming the acoustic mass-law and a coincidence STL dip at $2071 \mathrm{~Hz}$. Compared to the LRM plate, a wider STL peak and dip are obtained for the same mass addition. The STL peak does not occur inside or near the SB, but in a frequency range with free bending wave propagation. Contrary to the vibration attenuation inside the SB for a point force excitation, no peak STL enhancement is obtained inside the SB. The STL before the STL peak and after the STL dip also tends to the $1 \mathrm{~mm}$ homogeneous plate with mass density $1.8 \rho_{\text {host }}$ and $\rho_{\text {host }}$ respectively.

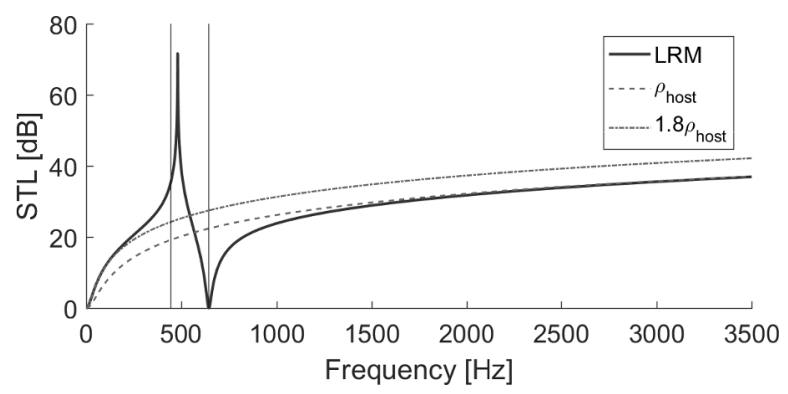

(a)

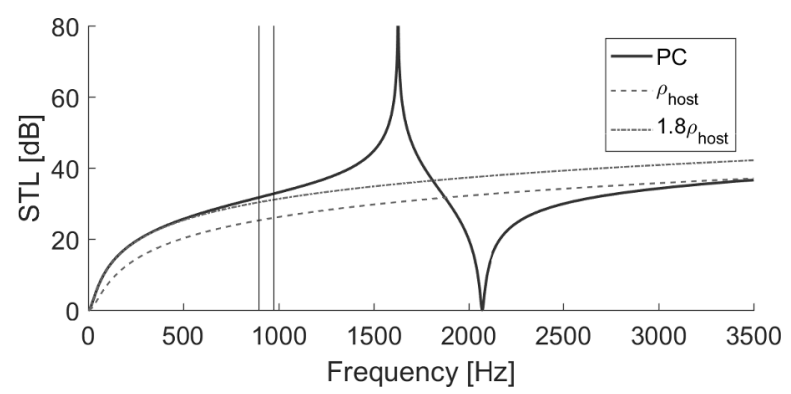

(b)

Fig. 4. Normal incidence STL of the infinite periodic LRM (a) and PC (b) plate, with SB (vertical lines).

The STL is further analyzed by means of the acoustic impedance of the LRM and PC plates (Figure 5). The impedance of the LRM plate shows an outspoken peak inside the SB, corresponding to the STL peak. For the PC plate, a different mechanism causes the interference SB and the impedance peak corresponding to the STL peak occurs outside the SB for the distributed acoustic excitation. The origin of the impedance and STL peak is analyzed using the RMS average out-of-plane displacement of the LRM and PC plate host structure and additions (Figure 6). The LRM plate resonator bases show a dip at $f_{\text {res }}$, indicating local vibration suppression. The sub-wavelength local vibration suppression leads to global vibration reduction with a dip at $480 \mathrm{~Hz}$, corresponding to the impedance and STL peak. The response of the resonator masses is lower than the host structure response after the SB. For the PC plate, no vibration reduction is found inside the SB. The minimum PC plate response at $1538 \mathrm{~Hz}$ does not correspond to the impedance and STL peak, nor does it indicate outspoken global vibration reduction. The response of the PC plate masses is also lower than the host structure response after the STL dip.

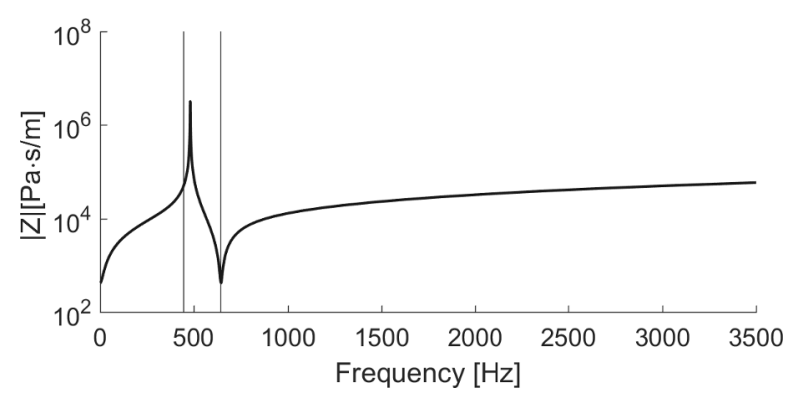

(a)

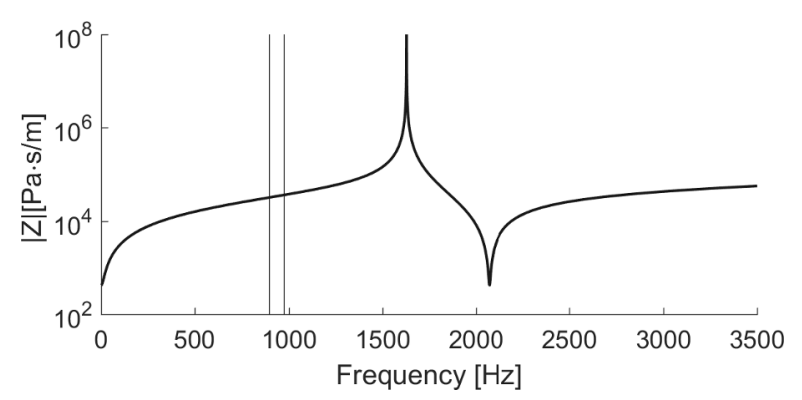

(b)

Fig. 5. Amplitude of the acoustic impedance of the infinite periodic LRM (a) and PC (b) plate for normal incidence, with SB (vertical lines).

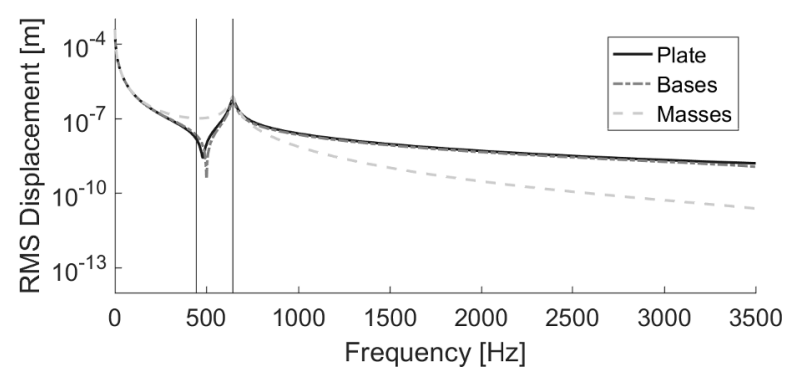

(a)

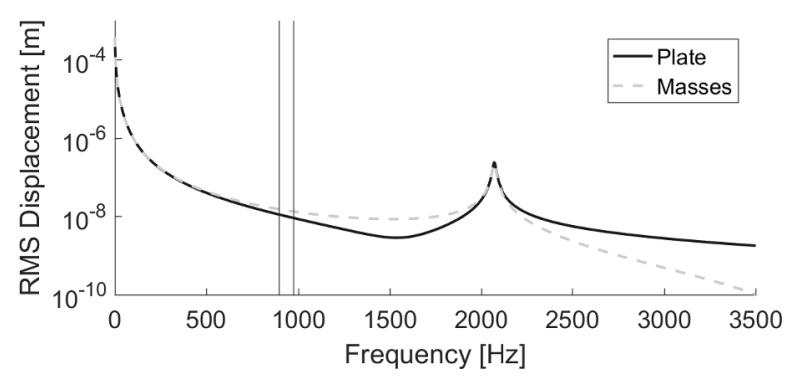

(b)

Fig. 6. RMS average displacement of plate, masses and resonator bases of the infinite periodic LRM (a) and PC (b) plate for normal incidence, with SB (vertical lines). 
To further analyze the STL of both infinite periodic plates, the sum of the out-of-plane plate velocities of the UCs is assessed (Figure 7). The impedance and STL peaks at $480 \mathrm{~Hz}$ for the LRM plate and at $1628 \mathrm{~Hz}$ for the PC plate correspond to dips in the net velocity amplitude. Consequently, the STL peak is related to a strongly reduced net out-of-plane response of the infinite periodic structure, which does not lead to efficient radiation. The vibration patterns of the LRM plate at $480 \mathrm{~Hz}$ and the PC plate at $1628 \mathrm{~Hz}$ are very similar, with a wavelength smaller than the acoustic wavelength (Figure 8). For the LRM plate, the vibration pattern is driven by the subwavelength local vibration suppression leading to global vibration reduction inside the SB. For the PC plate, this non-efficiently radiating sub-wavelength vibration pattern does not correspond to a minimum in vibration response (Figure 6b), but results from the vibro-acoustic interaction.

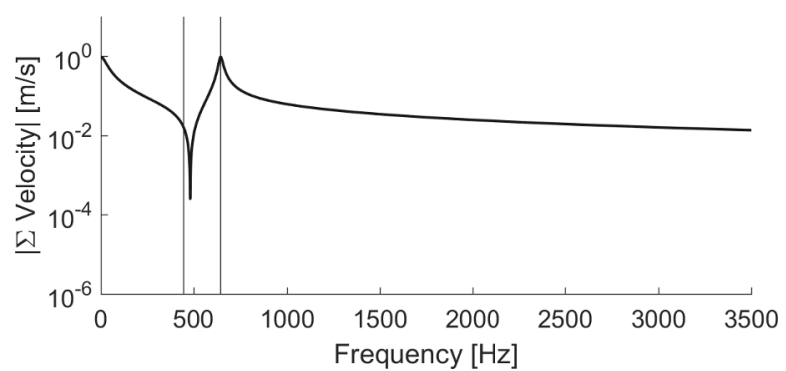

(a)

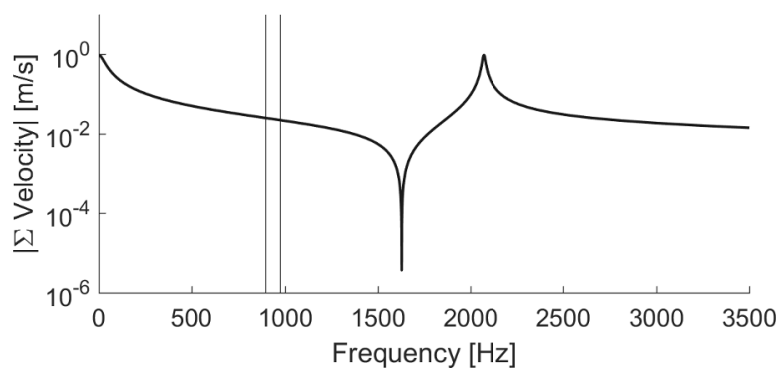

(b)

Fig. 7. Out-of-plane plate velocity sum amplitude of the UC of the infinite periodic LRM (a) and PC (b) plate for normal incidence, with SB (vertical lines).

\section{Finite plate STL}

To verify the potential of LRM and PC plates of a realizable size, the finite plate STL is analyzed for a $0.6 \times 0.45 \mathrm{~m}$ plate with simply supported BCs. This allows including finite-size effects and resonant STL behavior, apart from the non-resonant STL predicted for the infinite periodic plates. The normal incidence STL of the finite LRM plate agrees well with the infinite periodic structure STL (Figure 9). Outside the SB, structural modes are present, leading to resonant STL dips. Since no modes occur inside the $\mathrm{SB}$, the peak-dip behavior corresponds to the infinite periodic structure STL. The overall STL of the finite PC plate

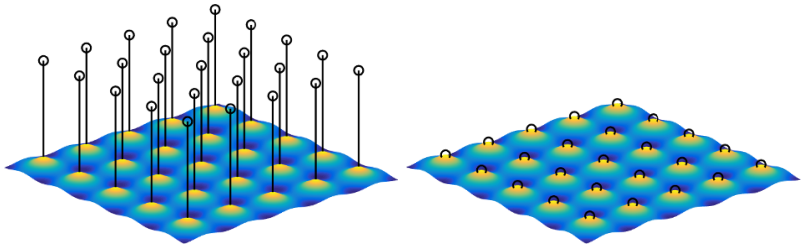

(a)

(b)

Fig. 8. Real displacement field of $5 \times 5$ UCs of the infinite LRM plate at $480 \mathrm{~Hz}$ (a) and PC plate at $1628 \mathrm{~Hz}$ (b) for normal incidence.

also corresponds to the infinite periodic structure STL, except around the predicted STL peak. No outpoken STL improvement is obtained inside the SB, apart from the absence of structural modes. The predicted STL peak for the infinite periodic structure is not obtained, as it occurs outside the SB and is reduced by structural mode STL dips.

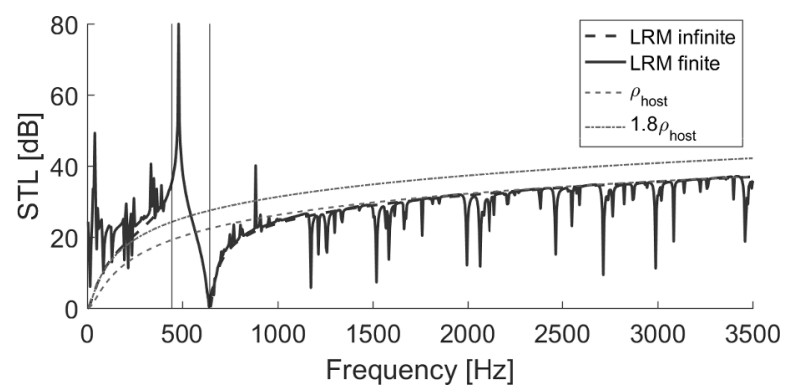

(a)

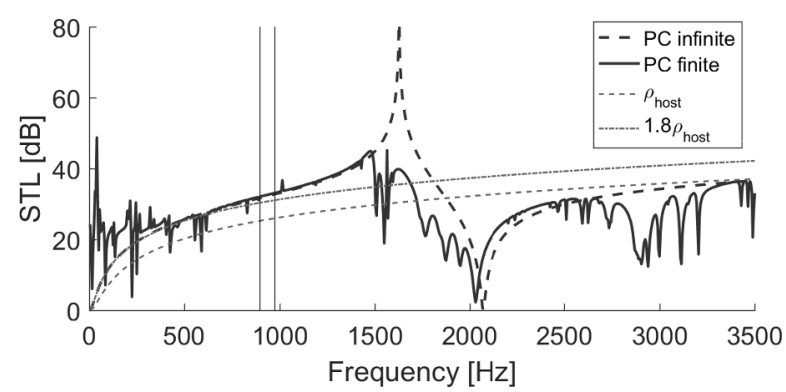

(b)

Fig. 9. Normal incidence STL of the infinite and finite LRM (a) and PC (b) plate, with SB (vertical lines).

The influence of structural modes is more outspoken in the diffuse field STL, calculated for a set of 12 oblique incident plane waves (Figure 10). The LRM plate STL is maintained and resembles the normal incidence STL. The diffuse STL of the PC plate is more affected by the structural modes, reducing the STL peak, while the STL in the following frequency range is strongly reduced.

The STL enhancement is further analyzed using the average acoustic impedance of the finite LRM and PC plates for normal incidence (Figure 11). The finite LRM 


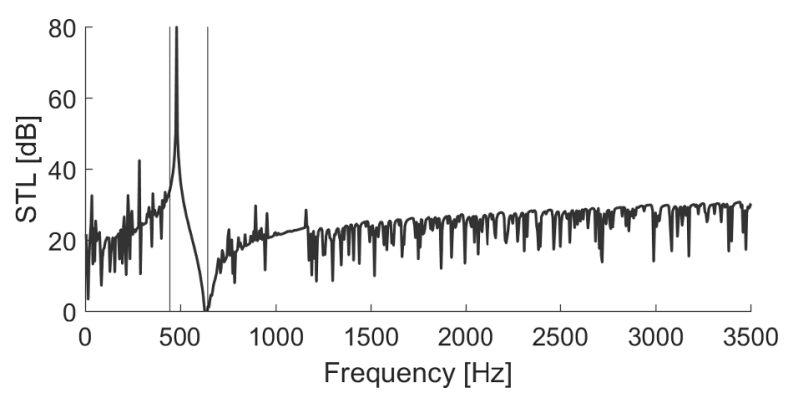

(a)

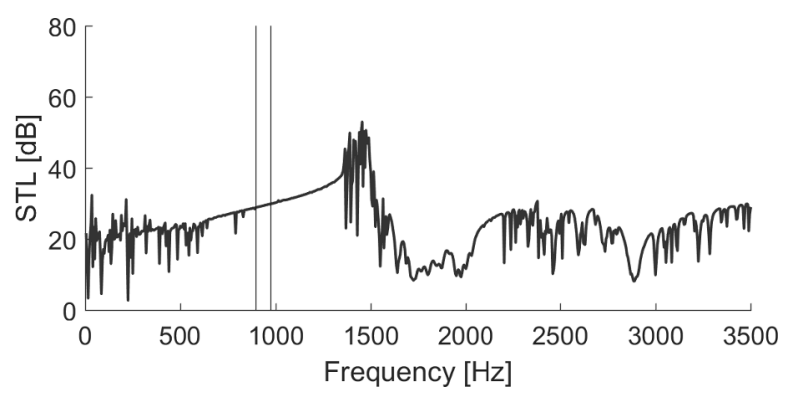

(b)

Fig. 10. Diffuse field STL of the finite LRM (a) and PC (b) plate, with SB (vertical lines).

plate impedance corresponds well with the infinite plate impedance (Figure 5), showing a peak at the peak STL frequency at $480 \mathrm{~Hz}$. The distributed sub-wavelength local vibration suppression leads to a global vibration reduction, resulting in an STL enhancement. The finite PC plate impedance differs from the infinite periodic structure impedance. The impedance does increase towards the predicted infinite periodic PC plate peak STL frequency, but is strongly reduced by the presence of structural modes. The RMS average out-of-plane displacement of the LRM and PC plate constituents again corresponds well to the infinite plate predictions, now including structural modes (Figure 12). The sub-wavelength local vibration suppression of the LRM plate, with inhibited resonator base motion at the tuned $f_{\text {res }}$, leads to global plate vibration reduction in the SB frequency range without the presence of structural modes. In the interference based SB of the PC plate no modes are present, but no global vibration reduction is obtained with respect to the distributed acoustic excitation. Around the predicted infinite PC plate STL peak, presence of structural modes is observed.

To gain insight in the STL behavior of both plates, the out-of-plane plate velocity sum of the LRM and PC plates is analyzed (Figure 13). For both plates, an outspoken dip occurs at the STL peak frequency predicted for the infinite periodic structure. As for the infinite periodic structure, the strongly reduced net out-of-plane velocity of the LRM plate is again related to the local vibration suppression which leads to global vibration reduction, resulting in the STL peak. The vibration pattern of the finite LRM plate at $480 \mathrm{~Hz}$ corresponds well to its infinite pe-

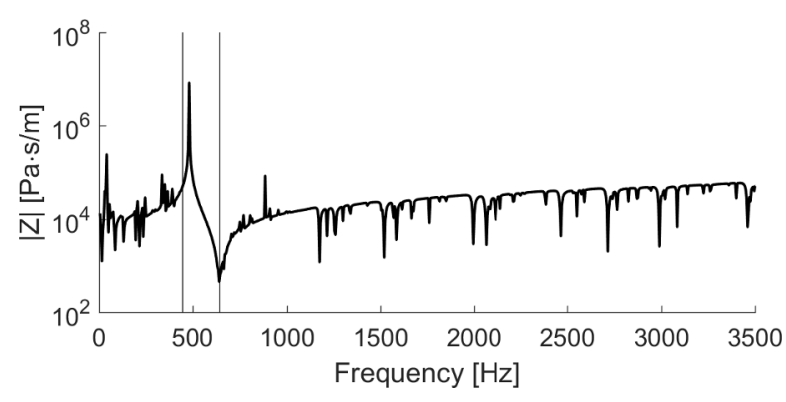

(a)

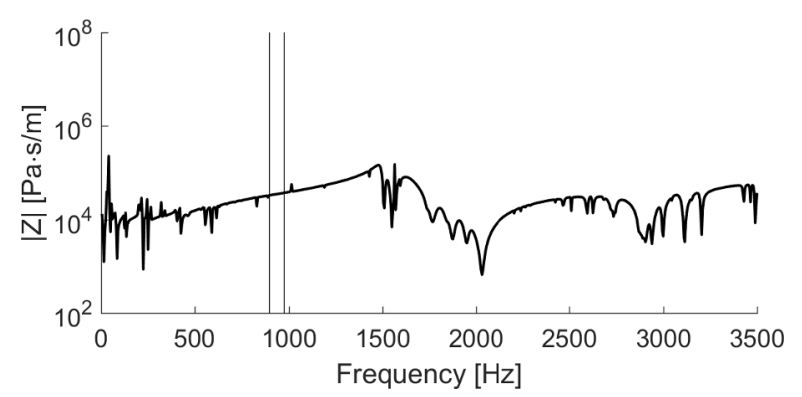

(b)

Fig. 11. Amplitude of the acoustic impedance of the finite LRM (a) and PC (b) plate for normal incidence, with SB (vertical lines).

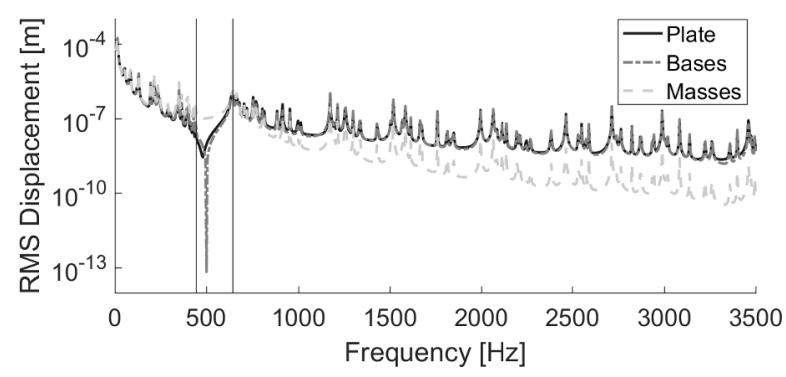

(a)

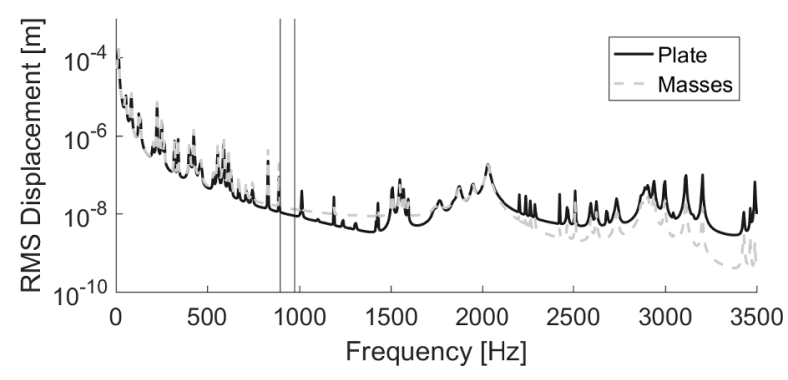

(b)

Fig. 12. RMS average displacement of plate, masses and resonator bases of the finite LRM (a) and PC (b) for normal incidence, with SB (vertical lines).

riodic counterpart, as no structural modes are allowed in the SB frequency range (Figure 14a). For the PC plate, 
however, the response at the minimum net out-of-plane velocity differs from its infinite periodic counterpart (Figure 14b). The non-efficiently radiating sub-wavelength vibration pattern enabling the STL peak enhancement for the infinite periodic structure is superimposed with structural modal behavior, since the STL peak of the infinite PC plate is predicted outside the SB. Consequently, the STL enhancement for the finite PC plate is not achieved.

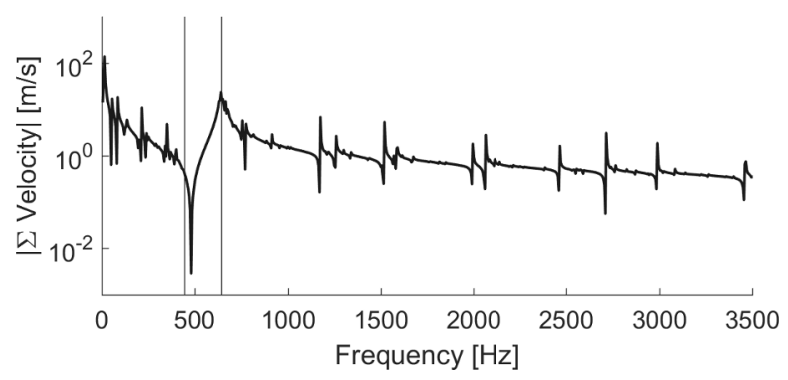

(a)

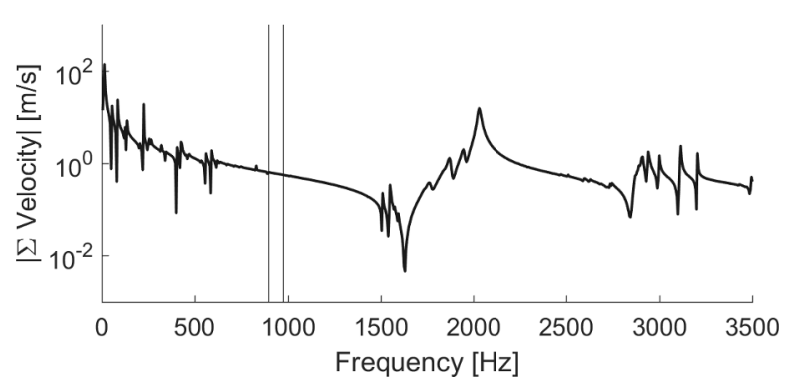

(b)

Fig. 13. Amplitude of the out-of-plane plate velocity sum of the finite LRM (a) and PC (b) plate for normal incidence, with SB (vertical lines).

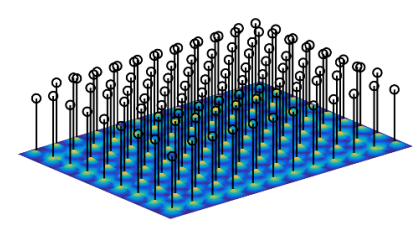

(a)

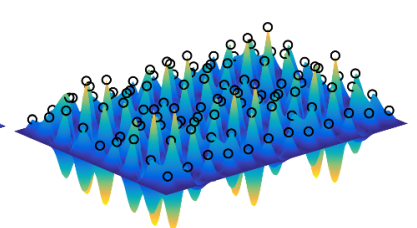

(b)
Fig. 14. Real displacement field of the finite LRM plate at $480 \mathrm{~Hz}$ (a) and PC plate at $1628 \mathrm{~Hz}$ (b) for normal incidence.

\section{Conclusion}

This paper investigates the potential of LRM and PC plates, with resonance and interference based bending wave SBs respectively, to obtain STL enhancements. An idealized LRM plate with periodic, sub-wavelength arranged spring-mass resonators and an idealized PC plate with periodically attached point masses are considered. Both structures exhibit bending wave SB behavior, leading to a zone of strong vibration attenuation and absence of structural bending modes for a finite plate subjected to a point force excitation. Mass-law outperforming STL improvements are predicted for both the infinite periodic LRM and PC plates. However, only the LRM plate leads to an STL enhancement inside the tuned SB, arising from the sub-wavelength vibration suppression leading to global vibration reduction. The STL enhancement of the infinite PC plate is predicted outside the SB and originates from a vibration pattern with strongly reduced net plate velocity. The STL of the finite LRM plate corresponds well to the infinite periodic LRM plate STL predictions. However, the STL enhancement predicted for the infinite periodic PC plate is not achieved, since the predicted STL peak occurs outside the SB and is reduced by the presence of structural modes.

The research of L. Van Belle and E. Deckers is funded by grants of the Research Foundation - Flanders (F.W.O.). The Research Fund KU Leuven is gratefully acknowledged for its support. This work is partially supported by Flanders Make, the strategic research centre for the manufacturing industry.

\section{References}

[1] M. I. Hussein, M.J. Leamy, M. Ruzzene, Appl. Mech. Rev. 66, 4 (2014)

[2] Y. Xiao, J. Wen, X. Wen, J. Sound. Vib. 331, 25 (2012)

[3] Y. Song, L. Feng, J. Wen, D. Yu, X. Wen, Compos. Struct. 128 (2015)

[4] P. Li, S. Yao, X. Zhou, G. Huang, G. Hu, J. Acoust. Soc. Am. 135, 4 (2014)

[5] J. Li, S. Li, Acta. Acust. United. Acust. 103, 5 (2017)

[6] C. Claeys, E. Deckers, B. Pluymers, W. Desmet, Mech. Syst. Signal Process. 70 (2016)

[7] C. Q. Howard, M. R. F. Kidner, Proceedings of Acoustics 2006 (2006)

[8] B. R. Mace, E. Manconi, J. Sound. Vib. 318, 4-5 (2008)

[9] E. Deckers, S. Jonckheere, L. Van Belle, C. Claeys, W. Desmet, J. Comput. Phys. 356 (2018)

[10] K. De Langhe, C. Moser, R. Boeykens, K. Kucukcoskun, Proceedings of Inter-Noise 2016 (2016)

[11] C. Claeys, P. Sas, W. Desmet, J. Sound. Vib. 333, 14 (2014) 